\author{
E.L. van der Kooi \\ J.S. Kalkman \\ E. Lindeman \\ J.C.M. Hendriks \\ B.G.M. van Engelen \\ G. Bleijenberg \\ G.W. Padberg
}

\title{
Effects of training and albuterol on pain and fatigue in facioscapulohumeral muscular dystrophy
}

Abstract Background We recently reported a randomised controlled trial on the efficacy of strength training and the $\beta 2$ adrenergic agonist albuterol in patients with facioscapulohumeral muscular dystrophy (FSHD). Strength training and albuterol appeared safe interventions with limited positive effect on muscle strength and volume. We concurrently explored the prevalence and the characteristics of pain and fatigue in the participating FSHD patients, because these are probably underreported but clinically relevant symptoms in this disorder. Next, we studied the effects of albuterol and strength training on pain, experienced fatigue, healthrelated functional status and psychological distress. Methods Sixtyfive patients were randomised to strength training of elbow flexors and ankle dorsiflexors or nontraining. After 26 weeks, albuterol

J.C.M. Hendriks, PhD

Department of Epidemiology \& Biostatistics

Radboud University Nijmegen Medical

Center

Nijmegen, The Netherlands (sustained-release, $8 \mathrm{mg}$ bid) was added in a randomised, doubleblind, placebo-controlled design. Outcomes comprised self-reported pain, experienced fatigue, functional status and psychological distress obtained with validated questionnaires at 52 weeks. Results Eighty percent of patients reported chronic persistent or periodic, multifocal pains. Thirtyfour percent of the participants were severely fatigued. Strength training and albuterol failed to have a significant effect on all outcomes. Conclusions Pain and fatigue are important features in FSHD. Strength training and albuterol do not have a positive or negative effect on pain, experienced fatigue, functional status and psychological distress.

Key words exercise therapy · albuterol ' pain ' fatigue . facioscapulohumeral muscular dystrophy

Rehabilitation Section

University Medical Center

Utrecht, The Netherlands 


\section{Introduction}

In descriptions of facioscapulohumeral muscular dystrophy (FSHD) pain and fatigue are rarely mentioned as important clinical features of the condition. However, a questionnaire-based survey in 270 French and one in 84 Dutch members of patient support groups indicated that pain is present in $50-75 \%$ of patients [6, 22]. Bushby et al. reported four adult patients with FSHD in whom pain was a presenting complaint and remained their most disabling symptom [10]. In a recent questionnaire-based survey, $61 \%$ of 139 Dutch FSHD patients were severely fatigued [19]. Their experienced fatigue severity was associated with the severity of functional impairments in daily life. Actually, pain and fatigue seem to be important symptoms in FSHD; and they are not only underreported, but probably also undertreated. In patients with slowly progressive neuromuscular diseases, such as FSHD, combinations of muscle weakness, pain, fatigue, problems with locomotion and body weight can lead to reduced physical activity and a sedentary lifestyle [26]. Physical inactivity has a negative influence on quality of life and health outcomes [26].

In FSHD the decline in muscle strength and mass is progressive over years and follows in general a recognizable sequence of muscle involvement. However, there is a large, unexplained, interindividual variability in rate of progression, even within families sharing the same mutation $[30,36,41]$. The variable course within families and the typical asymmetric weakness has led to the hypothesis that daily exertion might be responsible for disease progression $[9,18]$. Uncontrolled studies on the effects of strength training in neuromuscular disorders, although including only 13 FSHD patients, suggested a positive effect of strength training and did not point towards extra susceptibility for muscle overstrain $[1,25,28,39]$. The results of a pilot study and a subsequent randomised, controlled trial in FSHD patients with the $\beta 2$-adrenergic agonist albuterol were indicative for an anabolic effect that wears off with prolonged use [20, 21]. In animals and healthy persons the strength-increasing effect of $\beta 2$-adrenergic agonists can be augmented when it is administered in combination with resistance exercise $[11,14,16,42]$. We previously reported a randomised controlled trial in which we evaluated the efficacy of a moderate-intensity strength training program and albuterol in FSHD [35]. Strength training and albuterol were well tolerated and appeared safe interventions with limited positive effects on muscle strength and volume. Contrary to our expectation no synergistic effects between training and albuterol were detected; also no antagonistic effect was observed.
A strength training program could potentially induce muscle soreness caused by exercise-induced muscle damage or pain as a result of increased strain on the tendoskeletal apparatus. The imposed extra physical activity could lead to an increase in experienced fatigue caused by a physical overload, as well as a decrease caused by a better physical condition.

There is no information available on the effects of $\beta 2$-adrenergic agonists, such as albuterol, on muscle endurance or on experienced fatigue. Theoretically, albuterol could result in reduced fatigability via increased muscle strength and mass, but also in a decrease in muscle endurance by slow-to-fast muscle fibre type transformation [43]. Ideally, both interventions-strength training and albuterol-should result in less disability and a better quality of life.

The objectives of this study were: (1) to explore the prevalence, magnitude and other characteristics of pain and experienced fatigue in the group of FSHD patients participating in the previously reported, randomised controlled trial [35], and (2) to study the effects of albuterol and strength training on self-reported pain, experienced fatigue, functional status and psychological distress in these patients.

\section{Methods}

Sixty-five genetically confirmed, adult FSHD patients were randomised to strength training of elbow flexors and ankle dorsiflexors or non-training. After 26 weeks, albuterol (sustained-release, $8 \mathrm{mg}$ bid) was added in a double-blind, placebo-controlled design. Treatment was continued for another 26 weeks. For the effect of training the primary outcome measure was the maximum voluntary isometric strength (MVIC) of the elbow flexors and ankle dorsiflexors. Main secondary outcomes were muscle endurance and dynamic muscle strength of elbow flexors and ankle dorsiflexors. To evaluate the effect of albuterol the MVIC of eight large muscle groups, including elbow flexors and ankle dorsiflexors, was used as primary outcome measure. Main secondary outcome measures were muscle endurance and the total body skeletal muscle volume estimated by stereological CT method. For an extensive description of the subjects and interventions, the study design (see also Fig. 1), the test methods of the primary and main secondary outcomes, we refer to our previous publication [35]. The local ethics committee approved the study, and written informed consent was obtained from all subjects.

\section{Outcome measures}

The outcome measures concerning self-reported pain, experienced fatigue, health-related functional status and psychological distress were obtained with widely used, validated and reliable questionnaires and a self-observation list; all described hereafter. The questionnaires were sent to the participants' home three weeks before the baseline (week 0 ) and final visit (52 weeks), and handed in during these visits. The person who extracted the data from the questionnaires and made them suitable for statistical analysis was blinded for the assignment to both interventions. 


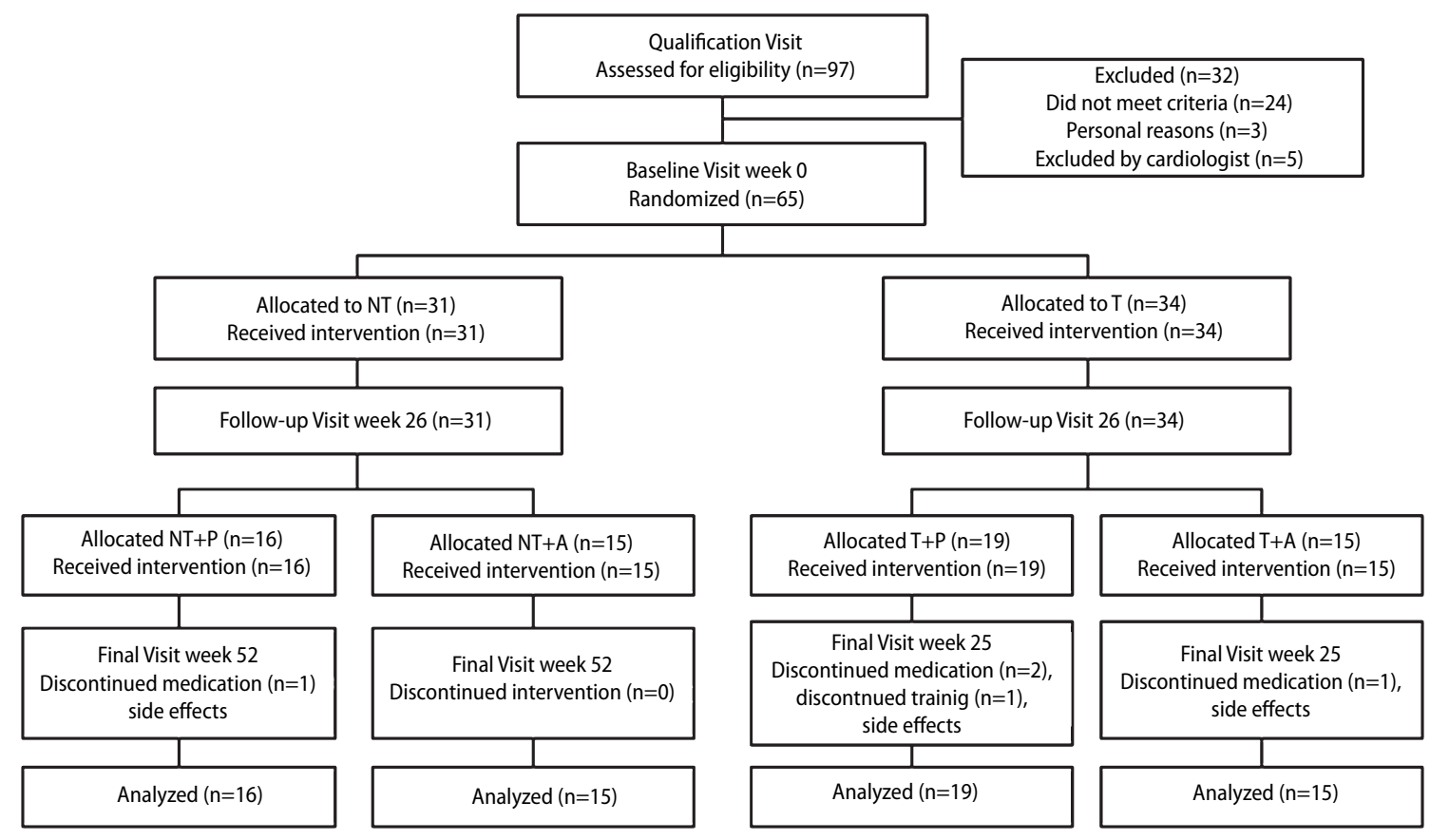

Fig. 1 Study design and flow of patients through each stage of the study. NT = non-training group, $T=$ training group, $P=$ placebo, and $A=$ albuterol

Pain

The McGill Pain Questionnaire (MPQ) was used for the assessment of different aspects of pain [27]. The MPQ is widely used, well validated and reliable [33,34]. The main outcome measure for pain was the magnitude of pain at that moment marked on a visual analogue scale (VAS); a VAS score of $0 \mathrm{~mm}$ indicating no pain, and $100 \mathrm{~mm}$ unbearable pain. In the MPQ patients were also asked to mark in the list of pain descriptors those adjectives that applied best to their pain. Finally, the MPQ included a whole body outline to indicate the distribution of the pain, and an inventory of the use of analgesics. In addition, the participants rated the intensity of their pain on a daily self-observation list during a two-week period. Pain severity was rated four times a day on a scale of 0 (no pain) to 4 (severe pain). The daily pain score could range from 0 to 16 , and the 14 daily pain scores were averaged into one Daily Observed Pain score (DOP) [31].

\section{Experienced fatigue}

The main outcome measure for experienced fatigue was the score on the fatigue severity subscale of the Checklist Individual Strength (CIS) [37]. In this questionnaire, the patient is asked to score the following four aspects of fatigue during the previous two weeks: fatigue severity ( 8 items), concentration problems (5 items), reduced motivation (4 items) and reduced activity (3 items). Each item is scored on a 7-point Likert scale. Higher scores indicate higher levels of fatigue, more concentration problems, low motivation and lower levels of activity. A CIS-fatigue subscale score equal to or higher than 35 identifies abnormal or severe fatigue [38]. The CIS questionnaire has good reliability and validity, including discriminative validity $[13,37,38]$. Experienced fatigue and self-reported activity levels were also measured with the daily self-observation list. Similar to the Daily Observed Pain score (DOP), a Daily Observed Fatigue score (DOF) and a Daily Observed Activity score (DOA) were calculated. A higher DOF score indicates more experienced fatigue, and a higher DOA score more self-reported activity [31].

\section{Functional status}

Health-related functional status was assessed using the original Sickness Impact Profile (SIP) $[8,17]$. Reliability and validity of the SIP have been evaluated extensively $[8,17]$. A total score of general disability (SIP-total) was calculated by adding the weights of items in the 12 categories or subscales: sleep/rest, emotional behaviour, body care/movement, household management, mobility, social interactions, ambulation, alertness behaviour, communication, work limitations, recreation/pastimes, and eating. The total SIP score ranges from 0 to 10,298 . A higher score means more disability.

\section{Psychological distress}

The Symptom Checklist-90 (SCL) was used to measure psychological distress [5]. This scale is widely used, and the reliability and validity, including discriminative validity are good [5]. The scale consists of 90 items scored on a 5-point Likert scale, and rates symptoms as anxiety, depression, sleeping problems and somatisation. The total score ranges from 90 to 450. A low total score reflects good psychological well-being, and higher scores indicate more distress. The Beck Depression Inventory - Primary Care (BDI-PC) was used as an additional outcome [7]. The BDI-PC was developed specifically for evaluating depression in medical patients by reflecting only the cognitive and affective symptoms of depression, while excluding somatic and performance items that might be related to medical problems (e.g. tiredness or fatigue, worries about physical problems). The BDI-PC is a 7-item questionnaire measuring depression on a 4-point scale ranging from 0 to 3 points per item. A score of 4 or more indicates a depression.

\section{Statistics}

To describe the study population at baseline the following methods or measures were used: (a) two-sided $t$-tests to test for statistically significant differences $(\alpha=0.05)$ between subgroups, in case of continuous variables; (b) $\chi^{2}$ test for nominal variables $(\alpha=0.05)$; 
and (c) Pearson coefficient $(r)$ for correlations. To retain power with these relatively small sample sizes, and because of the explorative nature of this descriptive part of the study, no adjustments were made for the multiple comparisons (at baseline).

The efficacy analyses were performed on an intention-to-treat basis, such that all randomised subjects were included in the analyses. A general linear mixed model was used to study possible differences between the four groups on the outcome measures, separately. The fixed independent variables were the study "visit" (0, 52 weeks), "training" (yes, no), "albuterol" (yes, no), "gender" (male, female), and "severity" ( $\geq 3,2$ trainable muscle groups). Per patient a random intercept was allowed. To detect differences between visits related to any of these variables all first order interaction terms between these variables and "visit" were included. Higher order interaction terms, except one second order term ("training" * "albuterol" * "visit") were excluded from the model, as they appeared to be not statistically significant (at the level of $\alpha=0.05$ ). The estimated means and effect sizes (difference in mean change from baseline analysed by intervention over all treatment groups) with $95 \%$ confidence intervals are presented. Tukey-Kramer adjustments for multiple comparisons were performed.

\section{Results}

\section{Subjects \& design}

The diagram in Fig. 1 shows the flow of patients through each stage of the study. Sixty-five patients were admitted to the study and randomised. The 25 females and 40 males had a mean age of $40 \pm 11$ and $37 \pm 10$ years, respectively. One patient stopped training, and four patients stopped using their study medication because of side effects [35]. All the participants completed their visits. Each patient had to complete six questionnaires for the baseline visit (week 0) and for the final visit (week 52). Only 10 of the 780 questionnaires were not handed in: five at baseline, and five at 52 weeks. The demographic and baseline characteristics of the patients are presented in Table 1.

\section{Baseline characteristics}

\section{Pain}

Thirteen out of 65 patients $(20 \%)$ reported on the MPQ to have no pain complaints at all. The other 52 patients had a VAS ranging from 0 to $83 \mathrm{~mm}$, with a mean VAS of $16 \mathrm{~mm}$. In the subgroup with pain these complaints were present for a mean duration of $85 \pm 69$ months. In $88 \%$ of these patients pain complaints had started gradually. Fifty percent of these patients reported periodic pains; in the other 50\% pain was constantly present with exacerbations. In $73 \%$ of patients with pain the complaints were always localized in the same body regions, with a mean of $4.3 \pm 2.7$ affected regions. Pain was most frequently present in the shoulder region, followed by the neck, lower back and lower leg (Table 2). Pain intensity was mostly described as mild to moderate, bearable to discomforting, annoying and tiring. Frequently used adjectives to characterize the pain were sore, stabbing, stiff and taut. Only seven patients (11\%) used analgesics regularly. Five of these patients used conventional first-line analgesics one to four times a week, and one patient daily. One patient used clonazepam once a week. Correlation between VAS and DOP scores were moderate $(r=0.65, \mathrm{p}<0.001)$. There was

Table 1 Baseline characteristics for the total study group, the pain subgroups and the fatigue subgroups

\begin{tabular}{|c|c|c|c|c|c|c|c|}
\hline & All patients $n=65$ & No pain $n=13$ & With pain $n=52$ & p-value* & Not sev. fatigued $n=43$ & Severely fatigued $n=22$ & $p$-value* \\
\hline \multicolumn{8}{|l|}{ Patient characteristics } \\
\hline Female, \% & 40 & 23 & 42 & 0.20 & 35 & 45 & 0.42 \\
\hline Age, years & $38 \pm 10$ & $37 \pm 12$ & $38 \pm 10$ & 0.66 & $38 \pm 11$ & $38 \pm 10$ & 0.79 \\
\hline \multicolumn{8}{|l|}{ Pain } \\
\hline VAS, $\mathrm{mm}$ & $12.9 \pm 17.1$ & $0 \pm 0$ & $16.1 \pm 17.7$ & 0.0018 & $9.0 \pm 14.0$ & $20.6 \pm 20.0$ & 0.008 \\
\hline DOP & $2.4 \pm 2.2$ & $0.4 \pm 0.7$ & $3.0 \pm 2.2$ & $<0.001$ & $2.0 \pm 2.0$ & $3.4 \pm 2.6$ & 0.013 \\
\hline \multicolumn{8}{|l|}{ Experienced fatigue } \\
\hline CIS-Fatigue & $28.8 \pm 12.1$ & $23.6 \pm 9.7$ & $30.1 \pm 12.4$ & 0.09 & - & - & - \\
\hline CIS_Fatigue $\geq 35, \%$ & 34 & 15 & 38 & 0.16 & - & - & - \\
\hline DOF & $3.4 \pm 2.3$ & $2.5 \pm 1.3$ & $3.7 \pm 2.3$ & 0.07 & $2.7 \pm 1.7$ & $5.1 \pm 2.1$ & $<0.001$ \\
\hline DOA & $6.1 \pm 2.0$ & $5.7 \pm 1.5$ & $6.3 \pm 2.1$ & 0.3 & $6.3 \pm 2.3$ & $5.8 \pm 1.4$ & 0.33 \\
\hline \multicolumn{8}{|l|}{ Functional status } \\
\hline SIP-Total & $708 \pm 612$ & $585 \pm 537$ & $739 \pm 630$ & 0.42 & $525 \pm 526$ & $1,091 \pm 594$ & $<0.001$ \\
\hline \multicolumn{8}{|l|}{ Psychological distress } \\
\hline SCL-Total & $117 \pm 29$ & $117 \pm 37$ & $115 \pm 24$ & 0.89 & $108 \pm 15$ & $131 \pm 37$ & $<0.001$ \\
\hline BDI-PC & $1.7 \pm 3.0$ & $2.1 \pm 4.8$ & $1.6 \pm 2.5$ & 0.63 & $0.9 \pm 1.7$ & $3.3 \pm 4.3$ & 0.0018 \\
\hline BDI-PC $\geq 4, \%$ & 16 & 8 & 18 & 0.39 & 7 & 33 & 0.008 \\
\hline
\end{tabular}

Values are means $\pm 1 S D$, unless specified differently

*Using $\chi^{2}$ test for comparing percentages and $t$-test for comparing means

VAS = visual analogue scale; DOP = Daily Observed Pain score; CIS = Checklist Individual Strength; DOF = Daily Observed Fatigue score; DOA = Daily Observed

Activity score; SIP = Sickness Impact Profile; SCL = Symptom Cheklist-90; BDI-PC = Beck Depression Inventory for Primary Care 
Table 2 Localization of pain as indicated on the bodily outline of the McGill Pain Questionnaire

\begin{tabular}{lllrll}
\hline Pain localization & Unilateral $n$ & Bilateral $n$ & Total $n$ & Total \% & Remarks \\
\hline Face & - & - & 3 & 5 & \\
Neck & - & - & 24 & 37 & \\
Shoulder girdle & $13^{*}$ & $20^{* *}$ & 33 & 51 & $*$ *8 right, 5 left ** rhomboid region \\
Upper arm & 5 & 8 & 13 & 20 & more frequent ventral side \\
Fore-arm, wrist, hand & 2 & 7 & 9 & 14 & including elbow \\
Abdomen, groin & - & - & 5 & 8 & \\
Back & - & - & 21 & 32 & mainly lower back \\
Hip region & 7 & 2 & 9 & 14 & \\
Thigh, upper leg & 6 & 8 & 14 & 22 & more frequent ventral side \\
Knee & 5 & 1 & 6 & 9 & \\
Lower leg, ankle, foot & 12 & 11 & 23 & 35 & ventral equals dorsal side \\
\hline
\end{tabular}

Each patient can have multiple localizations

$n=$ number of patients indicating pain on a specific localization

$\%=$ number of patients expressed as the percentage of the total study population no correlation between pain and activity as assessed with the DOP and DOA scores $(r=0.06, \mathrm{p}=0.66)$.

\section{Experienced fatigue}

Twenty-two patients (34\%) had a CIS-fatigue score equal to or higher than 35 indicating abnormal or severe fatigue. Also, the mean CIS-fatigue score of 28.8 was indicative for increased fatigue as compared to a group of healthy controls [37]. Mean scores on the subscales concentrations problems $(10.5 \pm 6.7)$, reduced motivation $(10.1 \pm 5.0)$, and reduced activity $(7.8 \pm 4.7)$ were similar or not significantly elevated as compared to healthy controls [37]. There was a moderate correlation between the CIS-fatigue score and the DOF score $(r=0.59$, $\mathrm{p}<0.001)$. Fatigue and activity as assessed with the DOF and DOA scores did not correlate $(r=-0.05$, $\mathrm{p}=0.73)$.

\section{Functional status and psychological distress}

Mean SIP-total score was $708.3 \pm 611.7$ implying relatively moderate sickness-related dysfunction in this group of FSHD patients [17]. The highest mean scores, indicating more problems, were in the categories sleep and rest, household management, ambulation, work limitations, and recreation and pastimes. Lower scores were found in the subscales emotional behaviour, body care and movement, mobility, social interactions, alertness behaviour, communication and eating.

Mean total and subscales scores of the SCL were within normal ranges, so there were no indications of abnormal psychological distress in the population studied. Sixteen percent of patients had a BDI-PC score of 4 or more, indicative of a depression. This percentage is not different from the prevalence of depression among patients with a medical illness in primary care settings [40].

\section{Comparison of patients with and without pain}

Baseline characteristics and values for the comparison of patients with and without pain are presented in Table 1. There was no relationship between the presence of pain and gender $\left(\chi^{2}=1.63, p=0.20\right)$. The mean VAS was not significantly different between males and females (females: $16.4 \pm 17.9 \mathrm{~mm}$, males: $10.7 \pm 16.3 \mathrm{~mm}, t$-test, $\mathrm{p}=0.3$ ). However, the mean DOP score was significantly higher for females (females $3.4 \pm 2.4$, males $1.8 \pm 1.9$, $t$-test, $\mathrm{p}=0.01$ ). Patients with and without pain were similar regarding their age. There was a weak correlation for both the VAS and the DOP scores and the age of patients, older patients having higher VAS and DOP scores (VAS: $r=0.28, \mathrm{p}=0.02$; DOP: $r=0.30, \mathrm{p}=0.02$ ).

The pain-free subgroup had a not significantly lower mean CIS-fatigue and DOF score (CIS-fatigue: $p=0.09$, DOF: $p=0.07)$. Fifteen percent of patients in the pain-free subgroup were severely fatigued as compared to $38 \%$ in the subgroup with pain $\left(\chi^{2}=2.46, \mathrm{p}=0.16\right)$. The mean DOA, SIP-total, SCLtotal, and BDI-PC scores did not differ significantly between both groups.

\section{Comparison of patients with and without severe fati- gue}

Baseline characteristics and values for the comparison of patients with and without severe fatigue are presented in Table 1. There was no relationship between the presence of severe fatigue and gender $\left(\chi^{2}=0.65\right.$, $\mathrm{p}=0.42)$. Also, the CIS-fatigue score was independent of gender (mean females: $30.7 \pm 11.2$; males: $27.6 \pm 12.6, t$-test, $\mathrm{p}=0.31$ ). Despite the moderate correlation between the CIS-fatigue score and the DOF score $(r=0.59, \mathrm{p}<0.001)$, there was a slightly, but significantly higher DOF score for females (mean females: $4.2 \pm 1.9$, males: $2.8 \pm 2.1, t$-test, $\mathrm{p}=0.01$ ). Patients in the severely fatigued subgroup had a similar age as compared to the not severely fatigued 
patients. CIS-fatigue and DOF scores showed no correlation with age (CIS-fatigue: $r=0.11, \mathrm{p}=0.39$; DOF: $r=0.09, \mathrm{p}=0.48$ ).

The severely fatigued subgroup had a significantly higher mean VAS and DOP score (VAS: $p=0.008$; DOP: $p=0.013)$. Nine percent of patients in the severely fatigued subgroup had no pain as compared to $26 \%$ of the not severely fatigued patients $\left(\chi^{2}=2.46\right.$, $\mathrm{p}=0.16)$. The mean DOA score did not differ significantly between both groups. The severely fatigued subgroup had a significantly higher SIP-total score ( $t$ test, $\mathrm{p}<0.001)$, and higher SCL-total ( $t$-test, $\mathrm{p}<0.001$ ), indicating more functional disability and more psychological distress. The BDI-PC score was also significantly higher in the severely fatigued patients $(\mathrm{p}=0.002)$. Thirty-three percent of patients in the severely fatigued subgroup had a BDI-PC score $\geq 4$, indicative of a depression, as compared to $7 \%$ of those who were not severely fatigued $\left(\chi^{2}=6.98\right.$, $\mathrm{p}=0.008)$.

\section{Interventions}

\section{Effect of strength training on pain}

Patients reported no notable muscle soreness to the physical therapist during his home visits. Eleven out of 34 patients in the training group reported increase of pain in neck and shoulder region at sometime during these home visits. Five mentioned a period with elbow complaints. The number of neck-shoulder and elbow complaints as assessed with the MPQ questionnaire did not differ between the training and non-training groups at baseline and at the final visit (Table 3).

After 52 weeks the main outcome measure for pain - the mean VAS-, and also the mean DOP scores of the training and non-training groups did not demonstrate significant changes (Table 4).

\section{Effect of strength training on experienced fatigue}

In general, patients reported to the physical therapist that their training-induced muscle fatigue lasted less than an hour. The training did not induce notable general fatigue, and they could carry out their daily

Table 3 Number of patients reporting neck, shoulder and elbow (pain) complaints by training group

\begin{tabular}{lccccc}
\hline \multirow{2}{*}{ Localization } & \multicolumn{2}{l}{ Non-training $(n=31)$} & & \multicolumn{2}{c}{ Training $(n=34)$} \\
\cline { 2 - 3 } \cline { 6 - 6 } & Week 0 & Week 52 & & Week 0 & Week 52 \\
\hline Neck-shoulder & 22 & 17 & & 21 & 19 \\
Elbow & 2 & 1 & 3 & 2 \\
\hline
\end{tabular}

activities normally afterwards. One patient stopped training because of recurring, training-related muscle soreness and fatigue. She had a second diagnostic work-up, revealing a mitochondrial myopathy as well as FSHD.

After 52 weeks the main outcome measure for experienced fatigue, the mean CIS-fatigue, did not demonstrate significant differences in changes between the training and non-training groups (Table 4). The mean DOF score of the patients in the training groups slightly decreased, while we observed a small increase in the non-training patients (effect size: -0.7 ; CI: -1.5 to $-0.01, \mathrm{p}=0.047$ ).

\section{Effect of albuterol on pain and fatigue}

Patients were called twice by a neurologist blinded for treatment allocation to check for initial and ongoing side effects of the study medication. Patients did not mention pain or fatigue as possible adverse effects.

The outcome measures for pain and fatigue of the albuterol and placebo groups did not change significantly between the baseline and final visit (Table 4).

\section{Effect of both interventions on functional status and psychological distress}

At the final visit the mean SIP-total, and it subscales, did not demonstrate relevant or significant changes for both the training and non-training groups, and the albuterol and placebo groups. Also, for all groups the mean SCL-total and the mean BDI scores did not change between the baseline and final visit (Table 4).

For all outcomes there were no differences between males and females. No synergistic effects between training and albuterol were detected; also no antagonistic effect was observed.

\section{Discussion}

Eighty percent of the participating FSHD patients reported chronic persistent or periodic pain with a low to moderate intensity, mostly characterized as sore, stabbing, stiff and taut. Pains were most frequently present around the shoulders, neck, lower back and lower legs. There was no relationship between the presence of pain and gender or age. However, if pain was present the reported intensity tended to be slightly higher for female and older patients. Patients with pain appeared to be more fatigued. The outcomes for functional status and psychological distress did not significantly differ between those with and without pain. 
Table 4 Results of strength training vs. non-training and albuterol vs. placebo on pain, experienced fatigue, functional status and psychological distress

\begin{tabular}{|c|c|c|c|c|c|c|}
\hline & \multicolumn{2}{|l|}{ Placebo $(n=35)$} & \multicolumn{2}{|l|}{ Albuterol $(n=30)$} & \multicolumn{2}{|l|}{ Effect size } \\
\hline & NT $(n=16)$ & $\mathrm{T}(n=19)$ & NT $(n=15)$ & $\mathrm{T}(n=15)$ & T vs. NT & A vs. $P$ \\
\hline \multicolumn{7}{|c|}{ Patient characteristic } \\
\hline Female, \% & 44 & 42 & 40 & 27 & & \\
\hline Age, years & $39 \pm 9$ & $36 \pm 9$ & $41 \pm 12$ & $36 \pm 11$ & & \\
\hline \multicolumn{7}{|l|}{ Pain } \\
\hline \multicolumn{7}{|l|}{ VAS, mm } \\
\hline Week 0 & $13.4(4.9-22.0)$ & $9.7(1.9-17.6)$ & $10.5(1.6-19.3)$ & $18.5(9.7-27.4)$ & $2.3(-6.6$ to 11.1$)$ & $1.5(-7.4$ to 10.4$)$ \\
\hline Week 52 & $9.0(0.4-17.6)$ & $12.1(4.2-20.0)$ & $12.0(3.1-20.9)$ & $17.9(9.0-26.8)$ & 0.6 & 0.7 \\
\hline \multicolumn{7}{|l|}{ DOP } \\
\hline Week 0 & $1.9(0.7-3.0)$ & $2.1(1.0-3.1)$ & $2.6(1.4-3.8)$ & $3.2(2.0-4.4)$ & $-0.7(-1.5$ to 0.1$)$ & $-0.7(-1.4$ to 0.2$)$ \\
\hline week 52 & $1.9(0.8-3.0)$ & $1.9(0.9-2.9)$ & $2.5(1.4-3.6)$ & $1.9(0.8-3.1)$ & 0.1 & 0.1 \\
\hline \multicolumn{7}{|c|}{ Experienced fatigue } \\
\hline \multicolumn{7}{|c|}{ CIS_Fatigue } \\
\hline Week 0 & $27.1(21.1-33.0)$ & $25.3(19.8-30.8)$ & $30.5(24.3-36.6)$ & $33.3(27.1-39.4)$ & $3.0(-2.0$ to 8.0$)$ & $-1.5(-6.5$ to 3.5$)$ \\
\hline Week 52 & $26.1(20.0-31.8)$ & $27.4(22.1-32.6)$ & $28.0(22.1-33.9)$ & $33.8(27.9-39.7)$ & 0.2 & 0.6 \\
\hline \multicolumn{7}{|l|}{ DOF } \\
\hline Week 0 & $3.6(2.5-4.7)$ & $3.1(2.0-4.1)$ & $2.7(1.7-3.8)$ & $4.2(3.1-5.4)$ & $-0.7(-1.5$ to -0.01$)$ & $0.1(-0.7$ to 0.8$)$ \\
\hline Week 52 & $3.7(2.7-4.6)$ & $2.7(1.8-3.6)$ & $3.1(2.1-4.1)$ & $3.6(2.6-4.6)$ & 0.047 & 0.9 \\
\hline \multicolumn{7}{|c|}{ Functional status } \\
\hline \multicolumn{7}{|c|}{ SIP-Total } \\
\hline Week 0 & $691(380-1,001)$ & $613(328-898)$ & $723(402-1,043)$ & $834(513-1,154)$ & $62(-105$ to 229$)$ & 20 (-148 to 187$)$ \\
\hline Week 52 & $565(227-903)$ & $530(220-840)$ & $599(247-951)$ & $790(441-1,139)$ & 0.5 & 0.8 \\
\hline \multicolumn{7}{|c|}{ Psychological distress } \\
\hline \multicolumn{7}{|c|}{ SCL-Total } \\
\hline Week 0 & $117(104-131)$ & $112(99-124)$ & $116(102-130)$ & $124(110-138)$ & $2(-6$ to 11$)$ & $3(-6$ to 12$)$ \\
\hline Week 52 & $111(101-121)$ & $106(97-116)$ & $110(100-121)$ & $123(112-133)$ & 0.6 & 0.6 \\
\hline \multicolumn{7}{|l|}{ BDI-PC } \\
\hline Week 0 & $1.9(0.4-3.4)$ & $0.7(-0.7-2.1)$ & $2.2(0.7-3.7)$ & $2.4(0.8-3.9)$ & $0.6(-0.5$ to 1.6$)$ & $-0.6(-1.6$ to 0.5$)$ \\
\hline Week 52 & $1.5(0.5-2.5)$ & $0.7(-0.2-1.6)$ & $1.1(0.1-2.1)$ & $1.9(0.9-2.9)$ & 0.3 & 0.3 \\
\hline
\end{tabular}

Values for age are means $\pm 1 \mathrm{SD}$

Mean values $(95 \% \mathrm{Cl})$ for all scores at the baseline (week 0) and final visit (week 52) presented by treatment group (i.e. non-training and placebo, training and placebo, non-training and albuterol, training and albuterol). As no statistically significant interactions between the two interventions (i.e. training vs. non-training; albuterol vs. placebo) could be detected, the effect sizes are presented by intervention. The effect sizes (95\% Cl; $\mathrm{p}$-value) represent the difference in mean change from baseline, analysed by intervention (i.e. training vs. non-training; albuterol vs. placebo) over all treatment groups.

NT = non-training group; $T=$ training group; $\mathrm{P}=$ placebo; and $\mathrm{A}=$ albuterol. VAS = visual analogue scale; DOP = Daily Observed Pain score; CIS = Checklist Individual Strength; DOF = Daily Observed Fatigue score; SIP = Sickness Impact Profile; SCL = Symptom Cheklist-90; BDI-PC = Beck Depression Inventory for Primary Care

Thirty-four percent of the patients were considered to be severely fatigued. There was no relationship between both the presence and the severity of fatigue, and gender or age. Severely fatigued patients had significantly more pain, more disability, more psychological distress, and more depressive feelings. However, our data also indicated relatively moderate sickness-related dysfunction and no indications of abnormal psychological distress in the total group of patients studied.

The two interventions, moderate-intensive strength training and albuterol, did not appear to have a clear positive or negative effect on pain, experienced fatigue, functional status or psychological distress.

The results of the Dutch and French surveys were rather similar with regard to relative frequency, pattern in time, and localization and other characteristics of pain $[6,22]$. Considering the high relative frequency of pain, it is surprising how little attention this complaint has received. For all, it raises questions about the pathophysiology of pain in this disorder. As suggested, part of the multifocal, often asymmetrical pains-especially those in the shoulder region and around the spine-can probably be attributed to postural problems [10]. Problems induced by the loss of muscle mass and strength, as pain was most frequently localized in body regions affected early and most severely in the course of the dystrophic process. However, a considerable proportion of our patients reported pain in the thighs and upper legs, and some patients even mentioned facial pains. We do not know if pain in these body parts is more myalgic in nature, as reported previously. Pain is also mentioned in relation to the onset and exacerbations of the disease $[10,30]$. Myalgic pains and periodic pains could be due to-a long debated-inflammatory component in the pathophysiology of FSHD [4]. Although inflammatory changes in muscle are a frequent histological feature in FSHD [4, 30], the results of metabolic 
investigations and muscle biopsies in the previously reported four patients with myalgic pains did not indicate any exceptional inflammatory response [10]. Moreover, the myalgic pains in these patients hardly responded to conventional first-line analgesics or anti-inflammatory therapy. Unfortunately, pain was not an outcome measure in a 12-week open-label pilot trial with prednisone in eight FSHD patients [32].

The presence of pain did not seem to have an important influence on disability and psychological distress; as opposed to the presence of severe experienced fatigue, which was clearly associated with more disability, more psychological distress and more depressive feelings. The proportion of patients with a possible depression was significantly higher in the severely fatigued subgroup as compared to the other patients. Severe fatigue could not be seen as merely a sign of depression, as $66 \%$ of the severely fatigued patients had normal scores on the depression inventory. However, it appears likely that in the state of depression fatigue related to FSHD is experienced as more severe. So, one should consider a depression in FSHD patients with excessive fatigue.

In a recent questionnaire-based survey $61 \%$ (CI: 53-69\%) of 139 Dutch FSHD patients were severely fatigued versus $34 \%$ (CI: $22-46 \%$ ) in our study [19]. Both studies used identical versions of the CIS questionnaire, and defined severe fatigue by the same cutoff score. The difference in the proportion of severely fatigued patients might be due to selection in both studies. The questionnaire-based survey was specifically aimed at problems associated with fatigue, potentially leading to an overestimation if nonresponders tended to be less fatigued. Our study might underestimate fatigue as a problem, as patients were only eligible if they were willing to train if allocated to the training group, and were able to walk independently, potentially excluding the most fatigued and most affected patients. Kalkman et al. also found an association between the experienced fatigue severity and functional impairments in daily life [19]. Recently, a 12-week low-intensity aerobic exercise program in eight FSHD patients improved their aerobic capacity without signs of muscle damage [29]. Self-reported fatigue did not change. Regrettably, this small study lacked a randomised controlled or casecontrolled design, and fatigue severity was not quantitated.

Experienced fatigue has been defined as an overwhelming sense of tiredness, lack of energy and feeling of exhaustion [23]. It is not the same as muscle weakness or muscle fatigability [23]. Muscle fatigability has been defined as the failure to sustain the force of muscle contraction, and is also called peripheral fatigue [12]. We believe that experienced fatigue in patients with a muscle disease is not merely caused by their muscle weakness and muscle fatigability alone. Central fatigue, characterized by an enhanced perception of effort and limited endurance of sustained physical and mental activities, might play a part as well [12]. Fatigue might even be an independent determinant of disability. We did not predefine a measure for disease severity, so at this moment we cannot answer the important question on the relations between experienced fatigue, the severity of the dystrophic process and functional disability.

We recently reported the primary and main secondary outcomes of this randomised controlled trial in which we demonstrated that in FSHD strength training and albuterol are well tolerated and safe interventions with limited positive effect on muscle strength and volume [35]. In the design of this study self-reported pain, experienced fatigue, functional status and psychological distress were predefined as explorative outcome measures.

The very limited positive effects of the strength training program on muscle strength and the absence of any detectable effects on experienced fatigue, functional status and psychological distress could reflect the inability of the diseased neuromuscular and cardiorespiratory system to respond with normal adaptations to the applied training stimulus. However, part of this lack of response could also be due to the specificity of the training $[15,24]$. The physiological adaptations to training are specific to muscle actions involved, speed of movement, range of motion, muscle groups trained, energy systems involved and intensity and volume of training [3]. This means that a training program with dynamic exercises increases dynamic strength more than isometric strength, and vice versa. This phenomenon of specificity of training also has implications for the sensitivity of the outcome measures: e.g. the positive effect of a dynamic strength training program may be captured by using a dynamic evaluation technique, and might be missed using an isometric strength measure, a functional test (e.g. walking), or a functional disability questionnaire. Of course, the secondary outcomes not demonstrating any relevant or significant changes may also be due to the small number of muscle groups trained. The American College of Sports Medicine recommends exercising eight to ten major muscle groups to come to an effective training stimulus [2]. All other recommendations to come to an effective, standardized, but safe and individualized exercise prescription were met. Based on our experience guardedly exercising eight to ten major muscle groups in neuromuscular patients does actually seem feasible. And finally, the FSHD population in this study turned out to have relatively moderate sicknessrelated dysfunction and no indications of abnormal 
psychological distress, so in retrospect perhaps one could hardly expect relevant changes.

However, in this trial no signs of overuse, such as a decline in strength measures or training-related increase in pain or fatigue, were seen. This is of major clinical importance, because these findings do not support the hypothesis of extra risk for muscle strain in FSHD. Still, an extra liability for overwork weakness in more severely affected FSHD patients is not excluded. Based on our experience we tell our FSHD patients that 'normal' participation in sports and work appears not to harm their muscles but there is insufficient evidence to establish that it offers benefit. And, that there is insufficient evidence for general prescription of exercise programmes in FSHD.

Pain and fatigue are indeed frequent and clinically relevant symptoms in FSHD. More work is necessary to understand their pathophysiology, to study their relation with disease severity and functional disability, and to explore possible treatment strategies. Now that concise strength training of a few muscle groups has proven safe, a study on the effect of more comprehensive training programs designed to target goals more meaningful to these patients seems rational. And, although albuterol (SR capsules, $8 \mathrm{mg}$ twice daily) did not have any effect on fatigue, functional status, and psychological distress, the limited positive effect on muscle strength and volume, and the good tolerability are sufficient ground for studies exploring alternative dosing regimens, and combinations with other ergogenic medication.

Acknowledgements The study was supported by a government grant of the Health Research and Development Council of the Netherlands (ZON-MW), the Prinses Beatrix Fonds, the Dutch Public Fund for Neuromuscular Disorders (VSN), and the Dutch FSHD Foundation. The authors thank Ria te Winkel-Slotboom for data management and advice. The authors also thank Hexal AG, Holzkirchen, Germany, for generously providing the study medication.

\section{References}

1. Aitkens SG, McCrory MA, Kilmer DD, Bernauer EM (1993) Moderate resistance exercise program: its effect in slowly progressive neuromuscular disease. Arch Phys Med Rehabil 74:711715

2. American College of Sports Medicine Position Stand (1998) The recommended quantity and quality of exercise for developing and maintaining cardiorespiratory and muscular fitness, and flexibility in healthy adults. Med Sci Sports Exerc 30:975-991

3. American College of Sports Medicine Position Stand (2002) Progression models in resistance training for healthy adults. Med Sci Sports Exerc 34:364-380

4. Arahata $\mathrm{K}$, Ishihara $\mathrm{T}$, Fukunaga $\mathrm{H}$, et al. (1995) Inflammatory response in facioscapulohumeral dystrophy (FSHD): immunocytochemical and genetic analysis. Muscle Nerve 2:S56-S66

5. Arindell WA, Ettema JHM (1986) SCL90 , handleiding bij multidimensionele psychopathologie-indicator. Swets \& Zeitlinger BV, Amsterdam

6. Association Française contre les $\mathrm{My}$ opathies (AFM) (1998) La monographie Myoline 'dystrophie musculaire facio-scapulo-humérale'. Evry Cedex

7. Beck AT, Guth D, Steer RA, Ball R (1997) Screening for major depression disorders in medical inpatients with the Beck Depression Inventory for Primary Care. Behav Res Ther 35:785-791
8. Bergner M, Bobbit RA, Carter WB, Gilson BS (1981) The Sickness Impact Scale: development and final revision of a health status measure. Med Care 19:787-805

9. Brouwer OF, Padberg GW, van der Ploeg RJ, Ruys CJ, Brand R (1992) The influence of handedness on the distribution of muscular weakness of the arm in facioscapulohumeral muscular dystrophy. Brain 115:1587-1598

10. Bushby KMD, Pollitt C, Johnson MA, Rogers MT, Chinnery PF (1998) Muscle pain as a prominent feature of facioscapulohumeral muscular dystrophy (FSHD): four illustrative case reports. Neuromuscul Disord 8:574-579

11. Caruso JF, Signorile JF, Perry AC, et al. (1995) The effects of albuterol and isokinetic exercise on the quadriceps muscle group. Med Sci Sports Exerc 27:1471-1476

12. Chaudhuri A, Behan PO (2004) Fatigue in neurological disorders Lancet 363:978-988

13. Dittner AJ, Wessely SC, Brown RG (2004) The assessment of fatigue, a practical guide for clinicians and researchers. J Psychosom Res 56:157170

14. Dupont-Versteegden EE (1996) Exercise and clenbuterol as strategies to decrease the progression of muscular dystrophy in mdx mice. J Appl Physiol 80:734-741
15. Häkkinen K (1994) Neuromuscular adaption during strength training, aging, detraining, and immobilization. Crit Rev Phys Rehabil Med 6:161-198

16. Hayes A, Williams DA (1997) Contractile properties of clenbuterol-treated mdx muscle are enhanced by lowintensity swimming. J Appl Physiol 82:435-439

17. Jacobs HM, Lutik A, Touw-Otten FWMM, de Melker RA (1990) [The sickness impact profile; results of an evaluation study of the Dutch version]. Ned Tijdschr Geneeskd 134:1950-1954

18. Johnson EW, Braddom R (1971) Overwork weakness in facioscapulohumeral muscular dystrophy. Arch Phys Med Rehabil 52:333-336

19. Kalkman JS, Schillings ML, van der Werf SP, et al. (2005) Experienced fatigue in facioscapulohumeral dystrophy, myotonic dystrophy and HMSN-I patients. J Neurol Neurosurg Psychiatry 76:1406-1409

20. Kissel JT, McDermott MP, Natarajan R, et al. (1998) Pilot trial of albuterol in facioscapulohumeral muscular dystrophy. FSH-DY Group. Neurology 50:1402-1406

21. Kissel JT, McDermott MP, Mendell JR, et al. (2001) Randomized, double-blind, placebo-controlled trial of albuterol in facioscapulohumeral dystrophy. Neurology 57:1434-1440 
22. Koetsier CP (1997) [Pain related to FSH dystrophy. An underestimated problem? Results of an inquiry in the Netherlands]. Dutch Public Fund for Neuromuscular Disorders (VSN). Baarn

23. Krupp LB (2003) Fatigue. Butterworth Heinemann, Philadelphia

24. Lindeman E, Drukker J (1994) Specificity of strength training in neuromuscular disorders. J Rehabil Sci 7:1315

25. McCartney N, Moroz D, Garner SH, McComas AJ (1988) The effects of strength training in patients with selected neuromuscular disorders. Med Sci Sports Exerc 20:362-368

26. McDonalds CM (2002) Physical activity, health impairments, and disability in neuromuscular disease. Am J Phys Med Rehabil 81:S108-S120

27. Melzack R (1975) The McGill Pain Questionnaire: major properties and scoring methods. Pain 1:277-299

28. Milner-Brown HS, Miller RG (1988) Muscle strengthening through highresistance weight training in patients with neuromuscular disorders. Arch Phys Med Rehabil 69:14-19

29. Olsen DB, Orngreen MC, Vissing J (2005) Aerobic training improves exercise performance in facioscapulohumeral muscular dystrophy. Neurology 64:1064-1066
30. Padberg GW (1982) Facioscapulohumeral disease [thesis]. Leiden University, Leiden

31. Prins JB, Bleijenberg G, Bazelmans E, et al. (2001) Cognitive behaviour therapy for chronic fatigue syndrome: a multicentre randomised controlled trial. Lancet 357:841-847

32. Tawil R, McDermott MP, Pandya S, et al. (1997) A pilot trial of prednisone in facioscapulohumeral muscular dystrophy. FSH-DY Group. Neurology 48:46-49

33. Vanderiet $\mathrm{K}$, Adriaensen $\mathrm{H}$, Carton $\mathrm{H}$, Vertommen H (1987) The McGill Pain Questionnaire constructed for the Dutch language (MPQ-DV). Preliminary data concerning reliability and validity. Pain 30:395-408

34. van der Kloot WA, Oostendorp RA, van der Meij J, van den Heuvel J (1995) [The Dutch version of the McGill pain questionnaire: a reliable pain questionnaire]. Ned Tijdschr Geneeskd 139:669-673

35. van der Kooi EL, Vogels OJ, van Asseldonk RJ, et al. (2004) Strength training and albuterol in facioscapulohumeral muscular dystrophy. Neurology 63:702-708

36. van Deutekom JC, Wijmenga C, van Tienhoven EA, et al. (1993) FSHD associated DNA rearrangements are due to deletions of integral copies of a $3.2 \mathrm{~kb}$ tandemly repeated unit. Hum Mol Genet 2:2037-2042
37. Vercoulen JH, Swanink CM, Fennis JF, Galama JM, van der Meer JW, Bleijenberg G (1994) Dimensional assessment of chronic fatigue syndrome. J Psychosom Res 38:383-392

38. Vercoulen JH, Alberts M, Bleijenberg G (1999) [The Checklist Individual Strength (CIS)]. Gedragstherapie 32:131-136

39. Vignos PJ Jr, Watkins MP (1966) The effect of exercise in muscular dystrophy. JAMA 197:843-848

40. Whooley MA, Simon GE (2000) Managing depression in medical outpatients. N Engl J Med 343:1942-1950

41. Wijmenga C, Hewitt JE, Sandkuijl LA, et al. (1992) Chromosome 4q DNA rearrangements associated with facioscapulohumeral muscular dystrophy. Nat Genet 2:26-30

42. Wineski LE, von Deutsch DA, Abukhalaf IK, Pitts SA, Potter DE, Paulsen DF (2002) Muscle-specific effects of hindlimb suspension and clenbuterol in mature male rats. Cells Tissues Organs 171:188-198

43. Yang YT, McElligott MA (1989) Multiple actions of beta-adrenergic agonists on skeletal muscle and adipose tissue. Biochem J 261:1-10 